\title{
Performance Analysis of the Unary Coding aided SWIPT in a Single-User Z-channel
}

\author{
Jie $\mathrm{Hu}^{*}$, Mengyuan $\mathrm{Li}^{*}$, Kun Yang ${ }^{\dagger+}$ and Liangyuan $\mathrm{Liu}^{\ddagger}$ \\ ${ }^{*}$ School of Information and Communication Engineering, University of Electronic Science and Technology of \\ China, Chengdu, China, 611731 \\ $\uparrow$ School of Computer Science and Electronic Engineering, University of Essex, Colchester, U.K., CO4 3SQ \\ Zhongshan College, University of Electronic Science and Technology of China, Zhongshan, China, 528402 \\ Email: hujie@uestc.edu.cn, 201721010518@std.uestc.edu.cn, kunyang@essex.ac.uk, liuly1997@126.com
}

\begin{abstract}
Radio frequency (RF) signal based simultaneous wireless information and power transfer (SWIPT) has emerged as a promising technique for satisfying both the communication and charging requests of the massively deployed IoT devices. Different from the physical layer and the medium-access-control layer design for coordinating the SWIPT in the RF band, we study its coding-level control from the information theoretical perspective. Due to its practical implementation of the decoder and its flexibility on the codeword structure, the unary code is chosen as a potential joint information and energy encoder. By conceiving the classic Z-channel, the mutual information and the energy harvesting performance of the unary coding aided SWIPT transceiver is analysed. Furthermore, the optimal codeword distribution is obtained for maximising the mutual information, while satisfying the minimum energy harvesting requirement. Our theoretical analysis and the optimal coding design are demonstrated by the numerical results.
\end{abstract}

\section{INTRODUCTION}

Due to its flexibility and controllability, the radio frequency (RF) signal based wireless power transfer (WPT) can be exploited for remotely charging the massively deployed Internet of Things (IoT) devices [1] [2]. Therefore, coordinating the WPT and the conventional wireless information transfer (WIT) in the same RF band yields the emerging technique of simultaneous wireless information and power transfer (SWIPT) [3].

A great deal of efforts have been invested in SWIPT spanning from the PHY layer transceiver design [4] to the MAC layer resource allocation [5] and networking [6]. Surprisingly, the research on the information theoretical essence of the SWIPT has not become prosperous, since Varshney et al. [7] firstly studied the tradeoff between the maximum mutual information and the energy harvesting requirement in different channels. This seminal work demonstrated that the performance of the SWIPT can be controlled by adjusting the codeword structure, which provides the theoretical fundamental for the coding controlled SWIPT. Furthermore, Tandon et al. [8] divided a codeword into several sub-blocks for satisfying the receiver's real-time energy demands. The

The authors would like to thank the financial support of National Natural Science Foundation of China (NSFC), No. U1705263, that of the Sichuan Science and Technology Program, No. 2019YJ0194, and that of Zhongshan City Team Project, No. 180809162197874. The sponsorship of GF Innovative Research Program is also gratefully acknowledged. optimal structure of the codeword is found for maximising the mutual information in a symmetric channel. However, both of [7] and [8] only considered memoryless information source in their analysis but ignored the correlation among the bits in the output sequence of a practical encoder. Therefore, these works optimised the transmit probabilities of the binary bits in order to achieve the maximum SWIPT performance without considering a practical coding scheme.

The conventional source encoders generates equi-probable binary bits for maximising the codewords' capability of the information transmission. However, the codewords having equi-probable binary bits are only capable of carrying a fixed amount of energy. Therefore, the conventional source encoder cannot satisfy the diverse energy requests of the receiver. The constrained code [9] has a certain degree of freedom to adjust the codeword structure for satisfying the WPT requirement without sacrificing the WIT efficiency. As a typical constrained code, the run-length-limited (RLL) encoder was designed by Fouladgar et al. [10] for minimising the battery overflow/underflow probability at the receiver subject to the constraint on the achievable mutual information. Furthermore, unary code has been widely used for the source coding [11], [12] in wireless communication, due to its low encoding and decoding complexity. Specifically, the unary code was used by Babar et al. [12] for the joint throughput and dimming control in the visible light communication system. However, the SWIPT performance of the unary code in the RF band has not been studied from the information theoretical perspective.

Against this background, our novel contributions are summarised as below:

- We propose a practical unary coding aided SWIPT transceiver and analysed its performance from the information theoretical perspective. The correlation among the information bits generated by the unary encoder has been taken into account in the performance analysis.

- The optimal codeword distribution is found for maximising the attainable lower bound (infimum) of the mutual information in the classic single-user Z-channel, while satisfying the energy requirement of the receiver.

- The achievable information rate, the amount of the energy delivered to the receiver and the average codeword length are evaluated by the numerical results. 


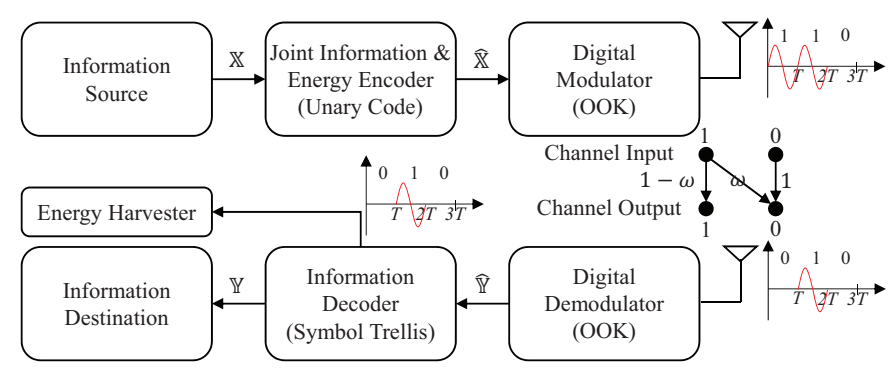

Fig. 1. The transceiver architecture of a single-user SWIPT system.

TABLE I

$K$-Level Unary Code

\begin{tabular}{llll}
\hline Messages & Message Prob. & Codewords & Codeword Prob. \\
\hline$X_{1}$ & $p\left(\mathcal{X}_{1}\right)$ & $\widehat{X}_{1}=0$ & $p\left(\widehat{X}_{1}\right)$ \\
$X_{2}$ & $p\left(X_{2}\right)$ & $\widehat{X}_{2}=10$ & $p\left(\widehat{X}_{2}\right)$ \\
$X_{3}$ & $p\left(X_{3}\right)$ & $\widehat{X}_{3}=110$ & $p\left(\widehat{X}_{3}\right)$ \\
$\vdots$ & $\vdots$ & $\vdots$ & $\vdots$ \\
$X_{M}$ & $p\left(X_{M}\right)$ & $\widehat{X}_{K}=\underbrace{11 \cdots 1}_{(K-1) \text { sign }} 0$ & $p\left(\widehat{X}_{K}\right)$ \\
& & & \\
\hline
\end{tabular}

The rest of the paper is organised as follows: the system model of the unary coding aided SWIPT transceiver is introduced in Section II, while its SWIPT performance is analysed in Section III. After providing the numerical results in Section IV, we finally conclude this paper in Section V.

\section{System Model}

The transceiver model of a single-user SWIPT system is illustrated in Fig.1. The transmitter is constituted by the following functional modules:

- Joint Information and Energy Encoderadopts the $K$-level unary coding for mapping $M$ messages $\mathcal{X}=\left\{\mathcal{X}_{1}, \cdots, \mathcal{X}_{M}\right\}$ randomly generated by the information source $\mathbb{X}$ onto $K$ codewords $\left\{\widehat{X}_{1}, \cdots, \widehat{X}_{K}\right\}$, where the $k$-th codeword $\widehat{X}_{k}$ has $(k-1)$ sign ' 1 ' followed by a sign ' 0 ', as portrayed in TABLE I. The resultant coded information source is denoted as $\widehat{\mathbb{X}}$. Given a specific message distribution $\mathbf{P}(\mathbb{X})=\left[p\left(\mathcal{X}_{1}\right), \cdots, p\left(\mathcal{X}_{M}\right)\right]$ of $\mathbb{X}$, we may obtain an arbitrary codeword distribution $\mathbf{P}(\widehat{\mathbb{X}})=\left[p\left(\widehat{\mathcal{X}}_{1}\right), \cdots, p\left(\widehat{\mathcal{X}}_{K}\right)\right]$ by exploiting the equation $\mathbf{P}(\widehat{\mathbb{X}})=\mathbf{P}(\widehat{\mathbb{X}}) \times \mathbf{P}(\widehat{\mathbb{X}} \mid \mathbb{X})$, where the matrix $\mathbf{P}(\widehat{\mathbb{X}} \mid \mathbb{X})=\left[p\left(\widehat{\mathcal{X}}_{k} \mid \mathcal{X}_{m}\right)\right]_{M \times K}$ is carefully chosen. The entry $p\left(\widehat{X}_{k} \mid X_{m}\right)$ is the probability of mapping the message $\mathcal{X}_{m}$ onto the codeword $\widehat{\mathcal{X}}_{k}$.

- Digital Modulator, which modulates the binary bit sequence on the amplitude and the phase of the carrier signals. Amplitude based digital modulator allocates different power to the arbitrary bit sequence. In order to highlight the impact of the codeword structure on the SWIPT performance, we adopt the on-off-keying (OOK) based modulator. The binary bit ' 1 ' is represented by the presence of the RF signal, while the binary bit ' 0 ' is represented by the absence of the RF signal. Therefore, the energy carried by a unary codeword is determined by the number of binary bit ' 1 '.
The effect of the channel attenuation between a transmitter and receiver pair can be modelled by the classic Z-channel, as illustrated in Fig.1:

- When a bit ' 0 ' is transmitted, the receive antenna only collects the noise. Since the noise power is far lower than that of the modulated RF signal, receiver may always make a correct detection. As a result, the output bit of the channel is surely ' 0 ', if a bit ' 0 ' is input to the channel. In this case, no energy can be harvested.

- When a bit ' 1 ' is transmitted, the power of the modulated RF signal is likely to be severely degraded and it may thus be overwhelmed by the noise, which results in an erroneous detection at the receiver and a complete energy loss. Therefore, the output bit of the channel is ' 0 ', which also represents no energy harvested. if the original bit ' 1 ' is input to the channel. This event occurs with a probability of $\omega$, which is also regarded as the crossover probability of the Z-channel.

- The RF signal carrying the bit ' 1 ' is not degraded a lot by the channel and its power is still significantly higher than the noise. The receiver makes a correct detection and the energy carried by the RF signal can be harvested by the receiver. Therefore, the output bit of the channel is ' 1 ', if the original bit ' 1 ' is input to the channel. This event occurs with a probability of $(1-\omega)$.

The Z-channel is sufficient to characterise the information and energy transmission of the OOK modulated RF signal between a single pair of the SWIPT transmitter and receiver, since no additional energy source exists in the system.

The following modules are implemented at the SWIPT receiver for the information decoding, as illustrated in Fig.1:

- Digital Demodulator, which demodulates the information carried by the received RF signal.

- Information Decoder, which decodes the received codeword in order to recover the original message. The symbol-level trellis can be invoked for efficiently decoding the unary encoded codeword [12], which is capable of avoiding the catastrophic error propagation.

- Information Destination receives the recovered message.

Similar to [7], [8], [10], we consider an ideal SWIPT receiver, in which the energy of the received RF signal flowing to the digital demodulator and the information decoder may not be lost. After the information decoding, the energy of the received RF signal can still be gleaned by the energy harvester.

\section{Information Theoretical Analysis}

\section{A. State Machine}

Given the $K$-level unary encoder, as illustrated in TABLE I, the codebook $\widehat{\mathcal{X}}$ consists of $K$ codewords, which are represented by $\widehat{X}=\left\{\widehat{X}_{1}, \widehat{X}_{2}, \cdots, \widehat{X}_{K}\right\}$. The probabilities of these codewords being transmitted can be denoted by $\mathbf{P}(\widehat{\mathbb{X}})=\left\{p\left(\widehat{X}_{1}\right), p\left(\widehat{X}_{2}\right), \cdots, p\left(\widehat{X}_{K}\right)\right\}$. A feasible codeword is arbitrarily chosen from the codebook $\widehat{\mathcal{X}}$ for conveying information and energy to the receiver. Sequentially transmitting a series of unary codewords generates an $\mathrm{N}$-bits binary sequence $\mathbf{X}_{N}=\left\{X_{1}, X_{2}, \cdots, X_{N}\right\}$. The correlation among the binary bits 


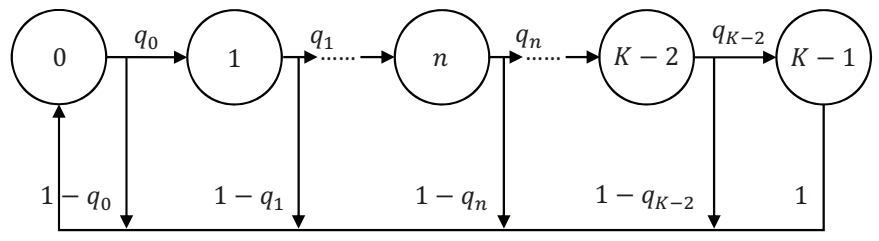

Fig. 2. The finite state machine of the unary code.

generated by the unary encoder can be modelled by a finitestate Markov chain, as portrayed in Fig.2.

State $n$ of this Markov chain represents that the unary coded information source $\widehat{\mathbb{X}}$ has already output a run of bit ' 1 ' having a length of $m$ at the current instant, since $\widehat{\mathbb{X}}$ is in the process of sending an arbitrary codeword chosen from $\left\{\widehat{X}_{n+1}, \widehat{X}_{n+2}, \cdots, \widehat{X}_{K}\right\}$. By considering the codeword structure of TABLE I, if $\widehat{\mathbb{X}}$ is in the process of sending codewords $\left\{\widehat{X}_{n+2}, \cdots, \widehat{X}_{K}\right\}$, the next output bit is ' 1 '. The run-length of bit ' 1 ' 'is then updated to $(n+1)$, which results in the state transition from $n$ to $(n+1)$. The corresponding state transition probability is expressed as

Moreover, the state transition probability $q_{n}$ is a function of the codeword distribution $\mathbf{P}$, which is formulated as:

$$
q_{n}=\frac{p\left(\widehat{X}_{n+2}\right)+\cdots+p\left(\widehat{X}_{K}\right)}{p\left(\widehat{X}_{n+1}\right)+p\left(\widehat{X}_{n+2}\right)+\cdots+p\left(\widehat{X}_{K}\right)},
$$

for $0 \leq n \leq K-2$. The probability of the Markov chain transiting from state $n$ back to state 0 is thus $\left(1-q_{n}\right)$. Specifically, when the Markov chain stays at state $(K-1)$, it transits back to state 0 at the next instant for certain, since $(K-1)$ is the maximum allowable run-length of bit ' 1 ' for a $K$-level unary code, as shown in TABLE I.

All the state transition probabilities can be collected into the following matrix $\mathbf{Q}$ :

$$
\mathbf{Q}=\left[\begin{array}{llllll}
1-q_{0} & q_{0} & 0 & \cdots & 0 & 0 \\
1-q_{1} & 0 & q_{1} & \cdots & 0 & 0 \\
\vdots & \vdots & \vdots & \ddots & \vdots & \vdots \\
1-q_{K-2} & 0 & 0 & \cdots & 0 & q_{K-2} \\
1 & 0 & 0 & \cdots & 0 & 0
\end{array}\right]
$$

The stationary distribution of the Markov chain can be represented by a row vector $\pi=\left\{\pi_{0}, \pi_{1}, \cdots, \pi_{K-1}\right\}$, which can be obtained by solving the following system of linear equations:

$$
\left\{\begin{array}{l}
\pi \times \mathbf{Q}=\pi \\
\pi \times \mathbf{I}_{K \times 1}=1
\end{array},\right.
$$

where $\mathbf{I}_{K \times 1}$ is a $K \times 1$ column vector having all its entries equal to one. Both $\boldsymbol{\pi}$ and $\mathbf{Q}$ will be used for calculating the mutual information.

A state sequence $\mathbf{S}_{N}=\left\{S_{1}, \cdots, S_{N}\right\}$ can be mapped onto a bit sequence $\mathbf{X}_{N}=\left\{X_{1}, \cdots, X_{N}\right\}$, as portrayed in Fig. 3 . For an example of a 4-level unary code, a 6-states sequence $\mathbf{S}_{6}=\{1,2,0,1,2,3,0\}$ corresponds to a bit sequence $\mathbf{X}_{6}=$ $\{1,1,0,1,1,1,0\}$.

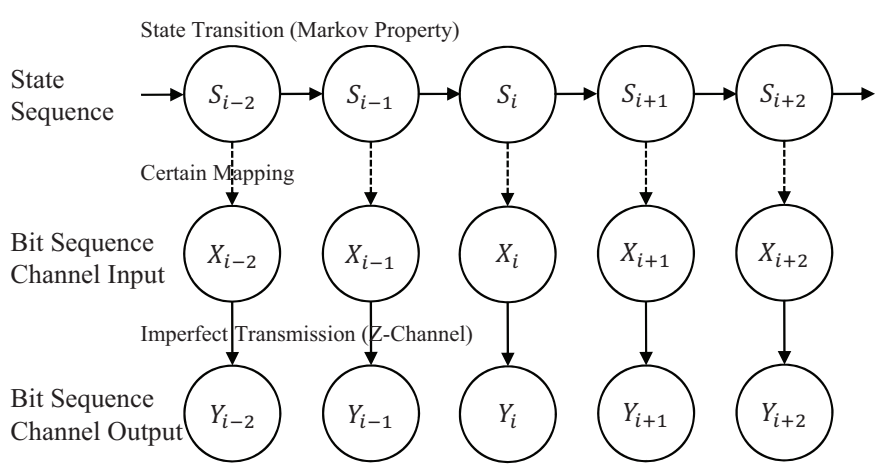

Fig. 3. The unary code's state transition, the certain mapping between the state sequence and the codeword as well as the random transmission between the channel input and the channel output. The subscript $i$ represents the $i$-th instant in the timeline.

\section{B. Mutual Information}

When the $N$-bit sequence $\mathbf{X}_{N}$ is input to the channel, the corresponding output bit sequence is $\mathbf{Y}_{N}=\left\{Y_{1}, Y_{2}, \cdots, Y_{N}\right\}$ after the imperfect transmission, as exemplified in Fig.3. Let us denote the coded information source (transmitter) as $\overline{\mathbb{X}}$ and the coded information destination (receiver) as $\widehat{\mathbb{Y}}$. Note that the unary coding aided transmitter is a stationary information source. The mutual information between $\widehat{\mathbb{X}}$ and $\widehat{\mathbb{Y}}$ represents the amount of information transmitted through the channel, which is formulated as

$$
I(\widehat{\mathbb{X}} ; \widehat{\mathbb{Y}})=H(\widehat{\mathbb{X}})-H(\widehat{\mathbb{X}} \mid \widehat{\mathbb{Y}}) .
$$

The information source's entropy $H(\widehat{\mathbb{X}})$ can be expressed as

$$
\begin{aligned}
H(\widehat{\mathbb{X}}) & =\lim _{N \rightarrow \infty} \frac{H\left(\mathbf{X}_{N}\right)}{N} \stackrel{(a)}{=} \lim _{N \rightarrow \infty} \frac{H\left(\mathbf{S}_{N}\right)}{N} \\
& \stackrel{(b)}{=} \lim _{N \rightarrow \infty} H\left(S_{N} \mid S_{1}, S_{2}, \cdots, S_{N-1}\right) \\
& \stackrel{(c)}{=} \lim _{N \rightarrow \infty} H\left(S_{N} \mid S_{N-1}\right) \\
& \stackrel{(d)}{=} H\left(S_{2} \mid S_{1}\right) .
\end{aligned}
$$

In Eq. (5), the equality (a) is derived due to the certain mapping between the bit sequence $\mathbf{X}_{N}$ and the state sequence $\mathbf{S}_{N}$, as exemplified in Fig.3. The equality $(b)$ is obtained by exploiting Theorem 4.2.1 of [13]. The equality $(c)$ is derived due to the Markov property of the state machine, while the derivation of the equality $(d)$ is based on the stationary property of the coded information source $\widehat{\mathbb{X}}$.

Observe from Fig.3 that the state sequence $\mathbf{S}_{N}$ is also correlated to the bit sequence $\mathbf{Y}_{N}$ of the channel output. As a result, in order to obtain the conditional entropy $H(\widehat{\mathbb{X}} \mid \widehat{Y})$ between the coded information source and the coded information destination, we firstly introduce the following pair of Lemmas for investigating the correlation between $\mathbf{S}_{N}$ and $\mathbf{Y}_{N}$ :

Lemma 1: The state $S_{i+1}$ at the $(i+1)$-th instant is independent of the channel output bits $\left\{Y_{1}, \cdots, Y_{i-1}, Y_{i}\right\}$ at the previous instants.

Proof: The Markov state machine transits from the state $S_{i}$ to $S_{i+1}$ due to the coding principle of the unary encoder. By contrast, the transition from the state $S_{i}$ to the bit $Y_{i}$ is induced 
by the imperfect information transmission in the Z-channel. Obviously, the transition process from the state $S_{i}$ to the state $S_{i+1}$ is physically uncorrelated to the transition process from the state $S_{i}$ to $Y_{i}$. By exploiting this uncorrelation, we may prove that the state $S_{i+1}$ is independent of $Y_{i}$. Please refer to our technical report [14] for the detailed proof.

Furthermore, we may prove that the channel output bits $\left\{Y_{1}, \cdots, Y_{i-1}\right\}$ are all uncorrelated to the state $S_{i+1}$. The reason is two-fold. Firstly, $\left\{Y_{1}, \cdots, Y_{i-1}\right\}$ only depends on the states $\left\{S_{1}, \cdots, S_{i-1}\right\}$, respectively. Secondly, the state $S_{i+1}$ only depends on $S_{i}$ but it is independent of $\left\{S_{1}, \cdots, S_{i-1}\right\}$.

Lemma 2: The state $S_{i}$ at the $i$-th instant is correlated with the channel output bits $\left\{Y_{i+1}, Y_{i+2}, \cdots\right\}$ at the subsequent instants. If the state transition probabilities satisfy $\left\{q_{n}=\right.$ $q \mid n=0,1, \cdots, K-1\}$, where $q \in(0,1)$ is a constant, $S_{i}$ is independent of $\left\{Y_{i+1}, Y_{i+2}, \cdots\right\}$.

Proof: The basic principle of proving this lemma is to find that the inequalities of $\left\{p\left(S_{i}=s_{i} \mid Y_{i+n}=y_{i+n}\right) \neq p\left(S_{i}=\right.\right.$ $\left.\left.s_{i}\right) \mid n=1,2, \cdots,\right\}$, where $s_{i} \in\{0,1, \cdots, K-1\}$ and $y_{i+n} \in\{0,1\}$. Furthermore, we also find the sufficient condition, when the equalities hold. Please refer to our technical report [14] for the detailed proof.

As a result, the conditional entropy $H(\widehat{\mathbb{X}} \mid \widehat{\mathbb{Y}})$ is derived as

$$
\begin{aligned}
& H(\widehat{\mathbb{X}} \mid \widehat{\mathbb{Y}})= \lim _{N \rightarrow \infty} \frac{H\left(\mathbf{X}_{N} \mid \mathbf{Y}_{N}\right)}{N}=\lim _{N \rightarrow \infty} \frac{H\left(\mathbf{S}_{N} \mid \mathbf{Y}_{N}\right)}{N} \\
& \stackrel{(e)}{=} \lim _{N \rightarrow \infty} \frac{1}{N}\left[H\left(S_{1} \mid Y_{1}, \cdots, Y_{N}\right)\right. \\
&\left.\quad+\sum_{i=2}^{N} H\left(S_{i} \mid S_{i-1}, \cdots, S_{1}, Y_{1}, \cdots, Y_{N}\right)\right] \\
& \stackrel{(f)}{=} \lim _{N \rightarrow \infty} \frac{1}{N}\left[H\left(S_{1} \mid Y_{1}, \cdots, Y_{N}\right)\right. \\
&\left.\quad+\sum_{i=2}^{N} H\left(S_{i} \mid S_{i-1}, Y_{i}, \cdots, Y_{N}\right)\right] \\
& \stackrel{(g)}{\leq} \lim _{N \rightarrow \infty} \frac{H\left(S_{1} \mid Y_{1}, \cdots, Y_{N}\right)+(N-1) H\left(S_{2} \mid S_{1}, Y_{2}\right)}{N} \\
&=\quad H\left(S_{2} \mid S_{1}, Y_{2}\right),
\end{aligned}
$$

where the equality $(e)$ is derived by exploiting the chain rule for entropy (Theorem 2.5.1 in [13]), whereas the equality $(f)$ is obtained by exploiting i) the Markov property of the state machine and ii) Lemma 1. According to Lemma 2, the state $S_{i}$ is correlated to the channel output bit sequence $\left\{Y_{i}, Y_{i+1}, \cdots, Y_{N}\right\}$, which yields the inequality of $(g)$ in Eq. (6).

By substituting Eqs. (5) and (6) into (4), the mutual information can be further expressed as

$$
\begin{aligned}
& I(\widehat{\mathbb{X}} ; \widehat{\mathbb{Y}}) \stackrel{(h)}{\geq} H\left(S_{2} \mid S_{1}\right)-H\left(S_{2} \mid S_{1}, Y_{2}\right)=I\left(S_{2} ; Y_{2} \mid S_{1}\right) \\
&=H\left(Y_{2} \mid S_{1}\right)-H\left(Y_{2} \mid S_{2}, S_{1}\right) \\
&=-\sum_{s_{1}} \sum_{y_{2}} p\left(y_{2}, s_{1}\right) \log _{2} p\left(y_{2} \mid s_{1}\right) \\
&+\sum_{s_{1}} \sum_{s_{2}} \sum_{y_{2}} p\left(y_{2}, s_{2}, s_{1}\right) \log _{2} p\left(y_{2} \mid s_{2} s_{1}\right) \\
& \stackrel{(i)}{=} \sum_{i=0}^{K-1} \pi_{i}\left\{H\left[q_{i}(1-\omega)\right]-q_{i} H(\omega)\right\} \triangleq I_{\mathrm{inf}}(\mathbb{X} ; \mathbb{Y}),
\end{aligned}
$$

where $H(x)=-x \log _{2}(x)-(1-x) \log _{2}(1-x)$ for $0 \leq x \leq 1$. The inequality $(h)$ of Eq. (7) is derived by substituting the upper bound $H\left(S_{2} \mid S_{1}, Y_{2}\right)$ of the conditional entropy $H(\widehat{\mathbb{X}} \mid \widehat{\mathbb{Y}})$. The equality $(i)$ of Eq. (7) is derived by exploiting the stationary distribution $\pi$ of the Markov state machine and its transition probability matrix $\mathbf{Q}$. The lower bound expressed in Eq. (7) is actually the infimum $I_{\mathrm{inf}}(\widehat{\mathbb{X}} ; \widehat{\mathbb{Y}})$ of the mutual information, because it is attainable according to Lemma 2. Since the entries in $\mathbf{Q}$ and those in $\pi$ are all functions of the codeword distribution $\mathbf{P}(\widehat{\mathbb{X}}), I_{\text {inf }}(\widehat{\mathbb{X}} ; \widehat{\mathbb{Y}})$ is also a function of $\mathbf{P}(\widehat{\mathbb{X}})$.

\section{Energy Harvested}

Without loss of generality, we assume that if bit ' 1 ' is received by the SWIPT receiver, it is capable of harvesting a single energy unit from the received RF signal. If bit ' 0 ' is received, the SWIPT receiver cannot harvest any energy. Therefore, the amount of energy arriving at the receiver depends on i) which codeword is transmitted, since different codewords carry different number of energy bit ' 1 ', and on ii) the imperfect transmission of the Z-channel, where energy bit ' 1 ' may be flipped to bit ' 0 ' resulting in the energy loss.

If the $K$-level unary encoder sends a codeword $\widehat{X}_{k}$, for $1 \leq$ $k \leq K$, it carries $(k-1)$ energy bits ' 1 '. After being transmitted by a Z-channel having a crossover probability $\omega$, only $j$ energy bits ' 1 ' are received, while the rest of $(k-1-j)$ bits ' 1 ', are flipped to ' 0 '. The probability of this event occurring is expressed as

$$
p_{(k-1) \rightarrow j}=\left(\begin{array}{c}
k-1 \\
j
\end{array}\right)(1-\omega)^{j} \omega^{k-1-j} .
$$

As a result, by averaging the effect of the Z-Channel, the expected energy harvested by the receiver from a single bit, when the codeword $\widehat{X}_{k}$ is transmitted, can be formulated as

$$
E\left(\widehat{\mathbb{Y}} \mid \widehat{X}_{k}\right)=\sum_{j=0}^{k-1} \frac{j \cdot p_{(k-1) \rightarrow j}}{k}=\sum_{j}^{k-1}\left(\begin{array}{c}
k-1 \\
j
\end{array}\right) \frac{j(1-\omega)^{j} \omega^{k-1-j}}{k} \text {. }
$$

By further averaging the transmission randomness of the $K$ level unary encoder, the expected energy gleaned by the receiver from a single bit can be expressed as

$$
\begin{aligned}
E(\widehat{\mathbb{Y}}) & =\sum_{k=1}^{K} p\left(\widehat{X}_{k}\right) E\left(\widehat{\mathbb{Y}} \mid \widehat{X}_{k}\right) \\
& =\sum_{k=1}^{K} \sum_{j}^{i-1}\left(\begin{array}{c}
k-1 \\
j
\end{array}\right) \frac{j p\left(\widehat{X}_{k}\right)(1-\omega)^{j} \omega^{k-1-j}}{k} .
\end{aligned}
$$

Furthermore, the average codeword length of the $K$-level unary code can be expressed as

$$
\bar{L}=\sum_{k=1}^{K} k \cdot p\left(\widehat{X}_{k}\right)
$$

\section{Problem Formulation}

When the $K$-level unary encoder is implemented, our optimal coding design problem can be then formulated as

$$
\begin{aligned}
& \text { Objective: } \max _{\mathbf{P}(\widehat{\mathbb{X}})} I_{\text {inf }}(\widehat{\mathbb{X}} ; \widehat{\mathbb{Y}}), \\
& \text { Subject to: } E(\widehat{\mathbb{Y}}) \geq E_{t h} .
\end{aligned}
$$




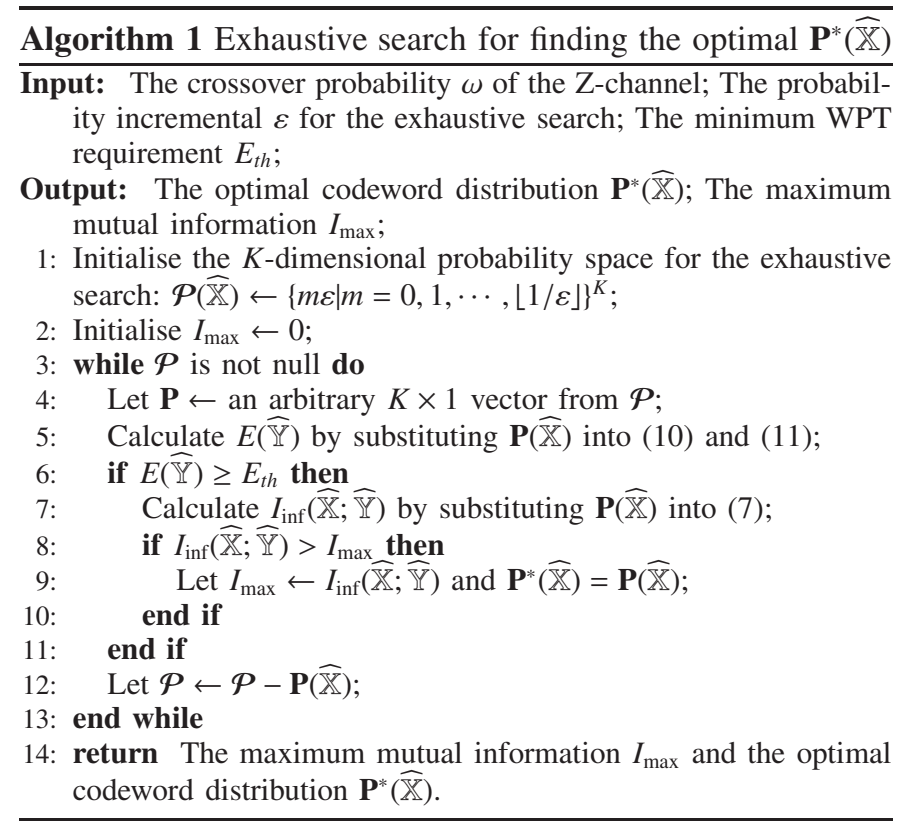

The objective of the optimisation problem (12) is to maximise the mutual information's infinimum $I_{\text {inf }}(\widehat{\mathbb{X}} ; \widehat{\mathbb{Y}})$ of Eq. (7) by finding the optimal codeword distribution $\mathbf{P}^{*}(\widehat{\mathbb{X}})$, while satisfying the constraint (12a) that the average energy $E(\widehat{\mathbb{Y}})$ harvested from a single bit should be higher than a minimum requirement $E_{t h}$.

However, since the calculation of $I_{\text {inf }}(\widehat{\mathbb{X}} ; \widehat{\mathbb{Y}})$ requires the stationary distribution $\pi$ and the transition probability matrix $\mathbf{Q}$, which may only be obtained by numerically solving the system of linear equations (3), the optimisation problem (12) cannot be solved in polynomial time, when we have a high-level unary encoder. As a result, an exhaustive search algorithm is proposed for obtaining the optimal codeword distribution $\mathbf{P}^{*}(\widehat{\mathbb{X}})$, as detailed in Algorithm 1 . The complexity of Algorithm 1 is $O\left(\lfloor 1 / \varepsilon\rfloor^{K}\right)$, which increases dramatically as we increase the searching incremental $\varepsilon$.

Note that if we ignore the constraint (12a), the problem (12) degenerates to a conventional mutual information maximisation problem. The resultant maximum mutual information is the channel capacity of the $K$-level unary coded system. The channel-capacity achieved codeword distribution can be denoted as $\mathbf{P}^{\dagger}(\widehat{\mathbb{X}})$.

\section{Numerical Results}

In this section, we characterise the impact of the minimum WPT requirement on both the maximum mutual information (representing the maximum WIT performance) and the corresponding average codeword length in the unary coded Zchannel. All the numerical results are obtained by setting the searching incremental of Algorithm 1 to $\varepsilon=0.01$.

We firstly investigate the SWIPT performance of the unary codes having different levels in Fig.4, where the Z-channel's crossover probability $\omega$ is fixed to 0.2. Observe from Fig.4(a) that the tradeoff between the maximum mutual information and the minimum WPT requirement does exist. When the

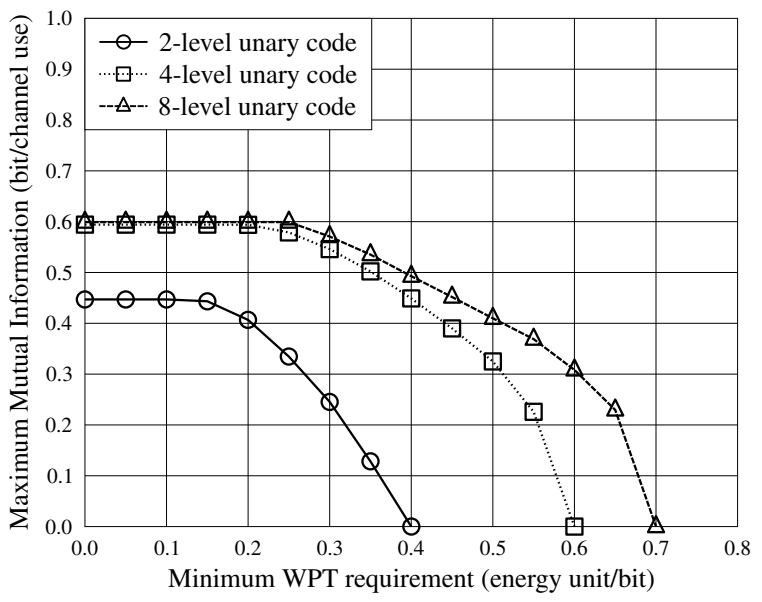

(a)

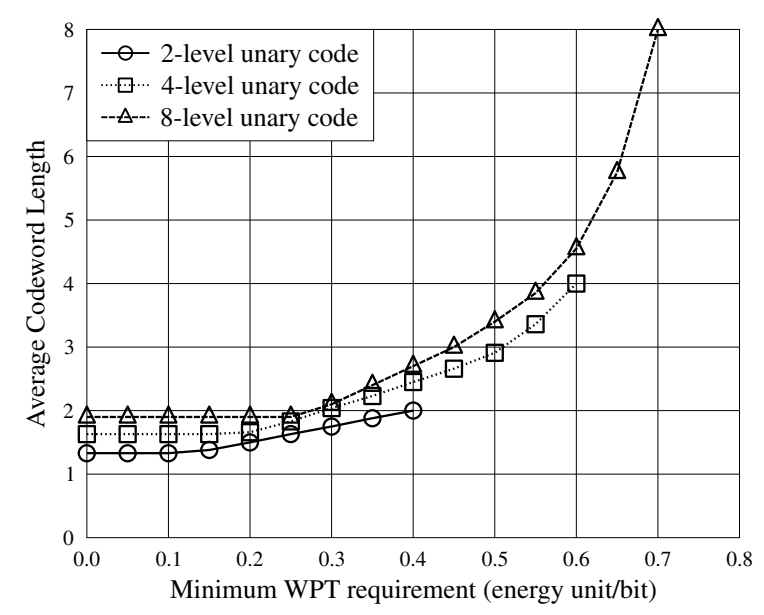

(b)

Fig. 4. The SWIPT performance of $(2,4,8)$-level unary codes having the crossover probability $\omega=0.2$ of the Z-channel: Minimum WPT requirement versus (a) maximum mutual information and versus (b) average codeword length .

WPT requirement is low, the codeword distribution $\mathbf{P}^{\dagger}(\widehat{\mathbb{X}})$ that achieves the channel capacity may readily satisfy the WPT requirement. As a result, the maximum mutual information, which is equal to the channel capacity, does not change as the WPT requirement increases. However, as the WPT requirement continuously becomes stringent, the channel capacity achieved codeword distribution $\mathbf{P}^{\dagger}(\widehat{\mathbb{X}})$ cannot satisfy the WPT requirement any more. Although the resultant codeword distribution $\mathbf{P}^{*}(\widehat{\mathbb{X}})$ maximises the mutual information subject to the WPT requirement, the degradation of the WIT performance is inevitable. The maximum WPT performance of the $K$ level unary code is achieved by always sending the $K$-th codeword $\mathcal{X}_{K}$, which has the highest number of energy bit ' 1 '. However, only sending a single codeword cannot convey any information. The maximum mutual information thus falls to zero. Furthermore, observe from Fig.4(a) that a higher level of unary code may convey both higher information and energy than the lower level counterpart. This is because a higher level unary code provides more degrees of freedom to encode information and to deliver energy. However, in practice, a high level unary code may incur the dramatic growth of the 


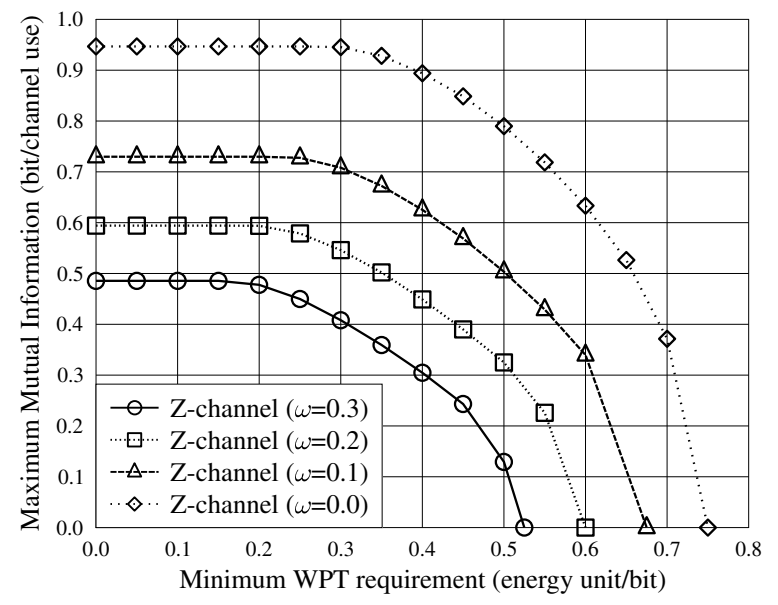

(a)

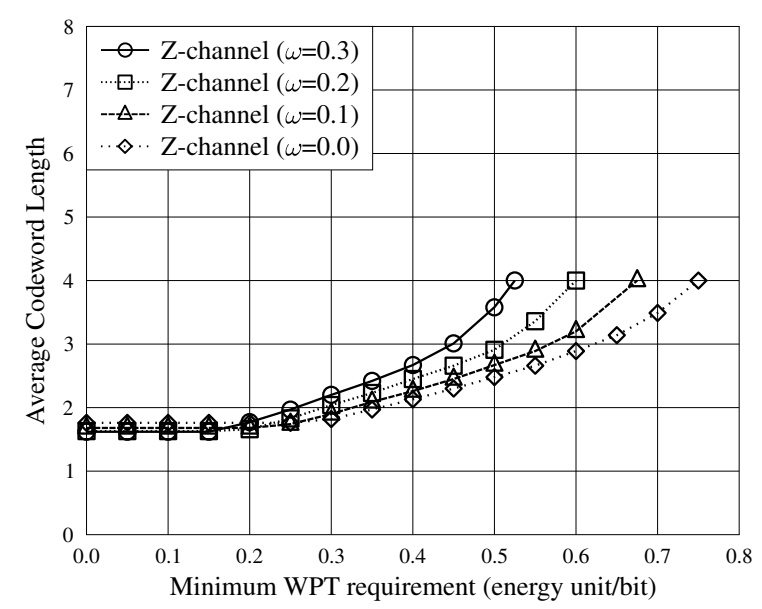

(b)

Fig. 5. The SWIPT performance of 4-level unary codes having the crossover probabilities $\omega=\{0.3,0.2,0.1,0\}$ of the Z-channel: Minimum WPT requirement versus (a) maximum mutual information and versus (b) average codeword length.

decoding complexity.

Observe from Fig.4(b) that as the WPT requirement becomes stringent, the codeword having a long length may be used more frequently, since it may carry more energy to fulfil the receiver's energy demand. In order to achieve the highest WPT requirement, the longest codeword has to be sent all the time. In this case, the average codeword length of the $K$-level unary code converges to $K$.

We then investigate the impact of the crossover probability $\omega$ on the SWIPT performance of the 4-level unary code. Observe from Fig.5(a) that as the crossover probability $\omega$ reduces, the 4-level unary code may achieve a better SWIPT performance. The impact of the crossover probability $\omega$ on the average codeword length is a little complicated. If the capacity-achieved codeword distribution $\mathbf{P}^{\dagger}(\widehat{\mathbb{X}})$ may satisfy the WPT requirement, which is characterised by the flat curves in the low WPT region of Fig.5(b), the average codeword length is reduced as the channel becomes worse. This is because any of the bit error in a codeword may result in the information loss. A longer codeword may thus incurs a higher information loss than its shorter counterpart. Therefore, when the channel becomes worse, the shorter codewords may be used more frequently in order to maximise the mutual information, which results in a shorter average codeword length. By contrast, when the capacity-achieved codeword distribution $\mathbf{P}^{\dagger}(\widehat{\mathbb{X}})$ cannot satisfy the WPT requirement, the long codewords have to be transmitted more frequently for counteracting the severe energy loss incurred by the channel in order to fulfil the energy demand of the receiver. As a result, in the high WPT region of Fig.5(b), as the channel becomes worse, the average codeword length increases.

\section{Conclusion}

We studied a unary code aided SWIPT transceiver in a classic single-user Z-channel. The unary encoder is modelled by a finite state Makrov chain, based on which the mutual information and the energy harvesting performance were analysed. The coding controlled SWIPT was formulated as a mutual information maximisation problem subject to the minimum energy harvesting requirement by finding the optimal codeword distribution. The numerical results demonstrated the tradeoff between the WIT and the WPT performance and the feasibility of the optimal coding controlled SWIPT.

\section{REFERENCES}

[1] K. Yang, S. Ou, H. Chen, and J. He, "A multihop peer-communication protocol with fairness guarantee for ieee 802.16-based vehicular networks," IEEE Transactions on Vehicular Technology, vol. 56, no. 6, pp. 3358-3370, Nov 2007.

[2] K. Yang, S. Ou, K. Guild, and H. Chen, "Convergence of ethernet pon and ieee 802.16 broadband access networks and its qos-aware dynamic bandwidth allocation scheme," IEEE Journal on Selected Areas in Communications, vol. 27, no. 2, pp. 101-116, February 2009.

[3] J. Hu, K. Yang, G. Wen, and L. Hanzo, "Integrated Data and Energy Communication Network: A Comprehensive Survey," IEEE Communications Surveys Tutorials, early access, August 2018.

[4] Y. Zhao, J. Hu, Z. Ding, and K. Yang, "Constellation Rotation Aided Modulation Design For the Multi-User SWIPT-NOMA," in 2018 IEEE ICC, May 2018.

[5] K. Lv, J. Hu, Q. Yu, and K. Yang, "Throughput maximization and fairness assurance in data and energy integrated communication networks," IEEE IoT Journal, vol. 5, no. 2, pp. 636-644, April 2018.

[6] Y. Zhao, D. Wang, J. Hu, and K. Yang, "H-AP Deployment for Joint Wireless Information and Energy Transfer in Smart Cities," IEEE Trans. on Vehi. Tech., early access, April 2018.

[7] L. R. Varshney, "Transporting Information and Energy Simultaneously," in 2008 IEEE ISIT, Jul. 2008, pp. 1612-1616.

[8] A. Tandon, M. Motani, and L. R. Varshney, "Subblock-Constrained Codes for Real-Time Simultaneous Energy and Information Transfer," IEEE Trans. on Info. Theory, vol. 62, no. 7, pp. 4212-4227, Jul. 2016.

[9] A. I. Barbero, E. Rosnes, G. Yang, and O. Ytrehus, "Constrained codes for passive RFID communication," in 2011 IEEE ITA, Feb. 2011, pp. $1-9$.

[10] A. M. Fouladgar, O. Simeone, and E. Erkip, "Constrained Codes for Joint Energy and Information Transfer," IEEE Trans. on Comm., vol. 62, no. 6, pp. 2121-2131, Jun. 2014.

[11] W. Zhang, Z. Song, M. F. Brejza, T. Wang, R. G. Maunder, and L. Hanzo, "Learning-aided unary error correction codes for nonstationary and unknown sources," IEEE Access, vol. 4, pp. 2408-2428, 2016.

[12] Z. Babar, M. A. M. Izhar, H. V. Nguyen, P. Botsinis, D. Alanis, D. Chandra, S. X. Ng, R. G. Maunder, and L. Hanzo, "Unary-Coded Dimming Control Improves ON-OFF Keying Visible Light Communication," IEEE Trans. on Comm., vol. 66, no. 1, pp. 255-264, Jan. 2018.

[13] T. M. Cover and J. A. Thomas, Elements of Information Theory, 2nd ed. John Wiley \& Sons, Inc., 2006.

[14] J. Hu, M. Li, and K. Yang, "Appendix of Performance Analysis of the Unary Coding aided SWIPT in a Single-User Z-channel," UESTC, Tech. Rep., October 2018. [Online]. Available: https://drive.google.c om/open?id=18pruJgW39rWB_A9FCqpbV8s81fmvwIf6 